OPEN ACCESS

Edited by:

Isabel Lastres-Becker, Autonomous University of Madrid,

Spain

Reviewed by:

Charles Robert Harrington,

University of Aberdeen,

United Kingdom

Alberto Rábano,

Fundacion Centro De Investigacion

De Enfermedades Neurologicas,

Spain

*Correspondence:

Thomas K. Karikari thomas.karikari@gu.se

${ }^{\dagger}$ These authors share first authorship

Received: 17 December 2019 Accepted: 27 February 2020

Published: 18 March 2020

Citation: Karikari TK, Thomas $R$ and Moffat KG (2020) The C291R Tau Variant Forms Different Types of Protofibrils. Front. Mol. Neurosci. 13:39. doi: 10.3389/fnmol.2020.00039

\section{The C291R Tau Variant Forms Different Types of Protofibrils}

\author{
Thomas K. Karikari ${ }^{1,2,3 * \dagger}$, Rachel Thomas ${ }^{1 \dagger}$ and Kevin G. Moffat ${ }^{1}$ \\ ${ }^{1}$ School of Life Sciences, University of Warwick, Coventry, United Kingdom, ${ }^{2}$ Midlands Integrative Biosciences Training \\ Partnership, University of Warwick, Coventry, United Kingdom, ${ }^{3}$ Department of Psychiatry and Neurochemistry, Institute of \\ Neuroscience and Physiology, The Sahlgrenska Academy, University of Gothenburg, Gothenburg, Sweden
}

Mutations in the MAPT gene can lead to disease-associated variants of tau. However, the pathological mechanisms behind these genetic tauopathies are poorly understood. Here, we characterized the aggregation stages and conformational changes of tau C291R, a recently described MAPT mutation with potential pathogenic functions. The C291R variant of the tau four-repeat domain (tau-K18; a functional fragment with increased aggregation propensity compared with the full-length protein), aggregated into a mix of granular oligomers, amorphous and annular pore-like aggregates, in nativestate and heparin-treated reactions as observed using atomic force microscopy (AFM) and negative-stained electron microscopy. On extended incubation in the native-state, tau-K18 C291R oligomers, unlike wild type (WT) tau-K18, aggregated to form protofibrils of four different phenotypes: (1) spherical annular; (2) spherical annular encapsulating granular oligomers; (3) ring-like annular but non-spherical; and (4) linear protofibrils. The ring-like tau-K18 C291R aggregates shared key properties of annular protofibrils previously described for other amyloidogenic proteins, in addition to two unique features: irregular/non-spherical-shaped annular protofibrils, and spherical protofibrils encapsulating granular oligomers. Tau-K18 C291R monomers had a circular dichroism (CD) peak at $\sim 210 \mathrm{~nm}$ compared with $\sim 199 \mathrm{~nm}$ for tau-K18 WT. These data suggest mutation-enhanced $\beta$-sheet propensity. Together, we describe the characterization of tau-K18 C291R, the first genetic mutation substituting a cysteine residue. The aggregation mechanism of tau-K18 C291R appears to involve $\beta$-sheet-rich granular oligomers which rearrange to form unique protofibrillar structures.

Keywords: MAPT mutations, tau C291R, corticobasal degeneration, granular oligomer, annular protofibril, linear protofibril, atomic force microscopy, transmission electron microscopy

\section{INTRODUCTION}

Tau protein is a product of the microtubule-associated protein tau (MAPT) gene located on chromosome 17q21 (Neve et al., 1986; Andreadis et al., 1992). MAPT is comprised of 16 exons, producing six tau isoforms in the adult human brain (Goedert et al., 1988, 1989). Tau has two significant components: the $\mathrm{N}$-terminus projection domain and the assembly domain covering

Abbreviations: AFM, atomic force microscopy; CBD, corticobasal degeneration; CD, circular dichroism; C291A, cysteine-291 substituted with alanine; C291R, cysteine-291 substituted for arginine; C291S, cysteine-291 substituted for serine; MTBR, microtubule-binding region; NaPhos, sodium phosphate; TBS-T, Tris-buffered saline plus Tween; TEM, transmission electron microscopy; WT, wild type. 
the microtubule-binding region (MTBR) and the C-terminus region (Andreadis et al., 1992; Andreadis, 2005). Variations in the number of $\mathrm{N}$-terminus domains $(0,1$ or 2$)$ and MTBR domains (3 or 4 repeats) are the defining features of different isoforms (Andreadis et al., 1992; Andreadis, 2005). Notably, alternative splicing of exon 10 affects the ratio of three- to four-repeat tau isoforms, changes in which have been linked to several tauopathies (Liu and Gong, 2008). Indeed, many MAPT mutations located in or around exon 10 have been reported from individuals affected by different genetic tauopathies, with over a dozen implicated in disease (Goedert and Jakes, 2005; Ghetti et al., 2015). However, until recently, none of the described mutations affected a cysteine residue. Each tau isoform has either one or two cysteine residues, depending on the number of repeat domains in the MTBR: four-repeat isoforms have two cysteine residues, at positions 291 and 322, whilst three-repeat isoforms have only cysteine-322. Therefore, cysteine-322 is ubiquitous to all tau isoforms whilst cysteine-291 is limited to four-repeat isoforms. Several studies have reported that the presence of the cysteine-291 residue is important for tau aggregation and that the cysteine-322 residue may be inhibitory to this process (Bhattacharya et al., 2001; Crowe et al., 2013; Soeda et al., 2015; Al-Hilaly et al., 2017).

In 2015, Marshall et al. (2015) identified a cysteine-modifying MAPT mutation altering cysteine-291 to arginine in a patient diagnosed with corticobasal degeneration (CBD) with apraxia of speech. This residue is sandwiched between two XSK tripeptide motifs (where $\mathrm{X}=\mathrm{Q}$ or G; Figure 1), changing it to arginine (Marshall et al., 2015). The disease relevance of this potential genetic form of $\mathrm{CBD}$ as yet cannot be confirmed since it has neither been observed at post-mortem nor traced to any relative of the patient. However, given the importance of cysteine residues, particularly cysteine291 , to specific physiological and pathophysiological functions of tau, including acetyltransferase activity and aggregation (Schweers et al., 1995; Cohen et al., 2013; Soeda et al., 2015; Al-Hilaly et al., 2017; Chen et al., 2018), we were interested in understanding how the C291R mutation might affect tau aggregation. Importantly, the core of tau filaments isolated from CBD patient brains consist of a broad range of amino acids (amino acids 274-380 of full-length tau) covering cysteine-291 and the lysine residues that immediately surround it (Zhang et al., 2020). These lysine residues immediately flanking cysteine-291 on either side (lysine-290 and lysine294) are thought to strengthen cysteine-291's disulfide bonding capacity, a property critical to tau protein's aggregation both in vitro and in vivo (Cisek et al., 2014). Indeed, the side chains of lysine-290 and lysine-294 are key components of an extra density structure within CBD filament folds (Zhang et al., 2020). We, therefore, hypothesized that substituting the hydrophobic cysteine-291 residue with arginine will generate a new stretch of basic amino acids that might lead to functional consequences on aggregation and conformation. In this study, we present the first biochemical characterization of tau C291R focusing on its step-wise aggregation stages, conformational and structural changes.

\section{MATERIALS AND METHODS}

\section{Cloning, Protein Expression and Purification}

We generated the pProEx-HTa-Myc-6 $\times$ His-K18 plasmid carrying the C291R mutation (TGT > CGT, the same codon change previously reported by Marshall et al., 2015) by site-directed mutagenesis using a WT tau-K18 plasmid as a template (Karikari et al., 2017, 2019a). Protein expression and purification were achieved using our previously-characterized recombinant tau production protocols (Karikari et al., 2017, 2019a,b; Hill et al., 2019). The purified His-tagged tau-K18 constructs were used directly in functional experiments since the His-tag does not appear to affect aggregation (Huseby et al., 2019). Protein concentration was estimated using a Bicinchoninic acid assay kit from G-Biosciences (\#786-570).

\section{Circular Dichroism (CD) Spectroscopy}

A Jasco J-815 CD spectropolarimeter was used to collect CD spectra on $22 \mu \mathrm{g} / \mathrm{ml}$ of purified tau-K18 WT and tau-K18 C291R, each diluted in $10 \mathrm{mM}$ sodium phosphate (NaPhos) buffer $\mathrm{pH}$ 7.4. The following conditions were used: cell path length $=1 \mathrm{~mm}$, data pitch $=0.1 \mathrm{~nm}$, response time $=1 \mathrm{~s}$, wavelength range $=190 \mathrm{~nm}-240 \mathrm{~nm}$, scan speed $=100 \mathrm{~nm} / \mathrm{min}$, and high-tension voltage $=\leq 550 \mathrm{~V}$. Averages of 10 spectral accumulations were analyzed.

\section{Heparin-Induced Aggregation Analysis}

A 2:1 mass concentration ratio reaction mix of WT or C291R tau-K18 to heparin was prepared using $0.8 \mathrm{mg} / \mathrm{ml}$ of tau-K18 and $0.4 \mathrm{mg} / \mathrm{ml}$ of heparin in a total volume of $20 \mu \mathrm{l}$. The mixture was incubated at $37^{\circ} \mathrm{C}$ without shaking for $48 \mathrm{~h}$ to form fibrils. Five microliters of each sample were spotted on Formvar carboncoated 300-mesh copper grids (\#AGS162-6, Agar Scientific, Stansted, UK) and allowed $2 \mathrm{~min}$ to bind. The unbound sample was removed by blotting with filter paper and $5 \mu$ l of uranyl acetate added for $2 \mathrm{~min}$. A JEOL JEM-2010 transmission electron microscope (TEM) was used to image the samples.

\section{Native-State Aggregation Time Course}

The tau-K18 preparations were incubated in $10 \mathrm{mM} \mathrm{NaPhos}$ buffer $\mathrm{pH} 7.4$ at $37^{\circ} \mathrm{C}$ for $0,24,72,168,216$ and $314 \mathrm{~h}$. At each time point $10 \mu \mathrm{l}$ aliquots were removed, snap-frozen and stored at $-80^{\circ} \mathrm{C}$ until further analysis by atomic force microscopy (AFM) as described below.

\section{AFM Analysis of Aggregation Stages}

Tau-K18 samples $(0.5 \mathrm{mg} / \mathrm{ml})$ from the aggregation time course experiment were spotted on to freshly-cleaved $11 \mathrm{~mm} \times 11 \mathrm{~mm}$ mica sheets (\#AGG250-3, Agar Scientific, Stansted, UK). Once dried, the unbound protein was washed away with sterile water and the mica sheet air-dried and analyzed in AC Air Topography (tapping) mode on an Asylum Research MFP3D-SA instrument. A scan size of $20 \mu \mathrm{m}$, a scan rate of $1 \mathrm{~Hz}$, set point of $590.49 \mathrm{mV}$, an integral gain of 3,x- and $y$ - offsets of 0 , points and gains of 256 and drive amplitude of $129.06 \mathrm{mV}$ were used. The Igor Pro 6.37 image processing and programming software was used for image processing. 


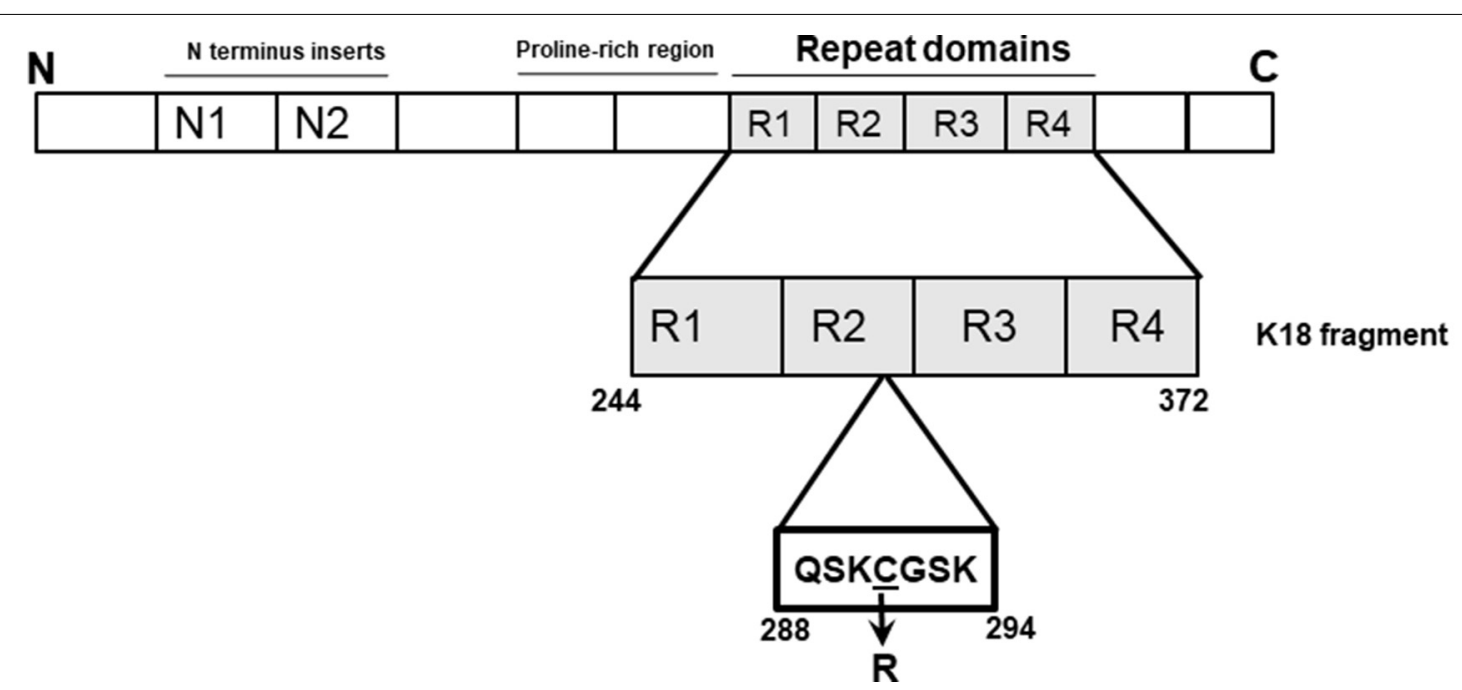

FIGURE 1 | Schematic illustration of the tau-K18 C291R construct used in this study. The microtubule-binding region of tau (amino acids 244-372 of full-length tau-441; also known as tau-K18) was cloned into a pProEx-HTa plasmid and the C291R mutation introduced by site-directed mutagenesis and sequence-verified. This genetic construct was transformed into BL21(DE3)* pRosetta Escherichia coli, expressed and purified following a previously-described protocol also used for preparing wild type tau-K18 (Karikari et al., 2017).
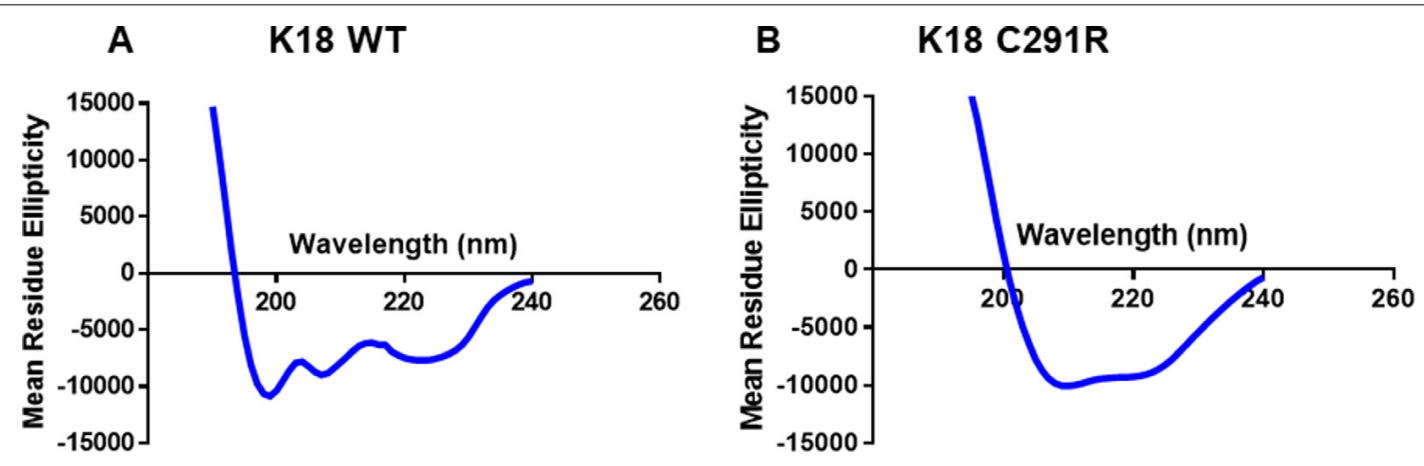

FIGURE 2 | Circular dichroism (CD) profiles of tau-K18 WT (A) and C291R (B) monomers. Each construct was diluted in $10 \mathrm{mM} \mathrm{NaPhos} \mathrm{buffer} \mathrm{pH} 7.4$ at $22 \mu \mathrm{g} / \mathrm{ml}$ final protein concentration and the secondary structure profiles analyzed as described in the "Materials and Methods" section.

\section{RESULTS}

\section{Secondary Structure Profile of Tau-K18 C291R}

Tau-K18 is generally unfolded but adopts $\beta$-sheet conformation with increasing aggregation (Kumar et al., 2014; Karikari et al., 2017). Specific MAPT mutations have distinct effects on this process by either increasing or decreasing the propensity to form $\beta$-sheet structures (Barghorn et al., 2000; Combs and Gamblin, 2012; Karikari et al., 2019a). To understand the possible effects of the C291R mutation on $\beta$-sheet formation, the secondary structure content of monomeric tau-K18 C291R and the WT were studied with CD spectroscopy. The experimental data in millidegrees were reconstructed in the Dichroweb database and the expected data (reconstructed minus experimental data) plotted in Figure 2. WT tau-K18 had a minimum peak of $\sim 199 \mathrm{~nm}$ whilst tau-K18 C291R had a peak at $\sim 210 \mathrm{~nm}$
(Figures 2A,B respectively), suggesting that the C291R variant has a higher propensity to adopt $\beta$-sheet conformation.

\section{Tau-K18 C291R Aggregates Into Amorphous and Spherical Structures, but Not Fibrils, Over $48 \mathrm{~h}$ in the Presence of Heparin}

As cysteine-291 is important for tau aggregation (Bhattacharya et al., 2001; Crowe et al., 2013; Soeda et al., 2015), we asked if the C291R mutation affects aggregation propensity. Each tau-K18 construct was mixed with a $50 \%$ mass concentration of heparin and incubated at $37^{\circ} \mathrm{C}$ without shaking for 48 h. Negative-stain TEM analysis showed that tau-K18 WT aggregated into fibrils (Figure 3A) in agreement with previous reports (Barghorn et al., 2000; Barghorn and Mandelkow, 2002; Karikari et al., 2017). These fibrils were 30-45 nm 


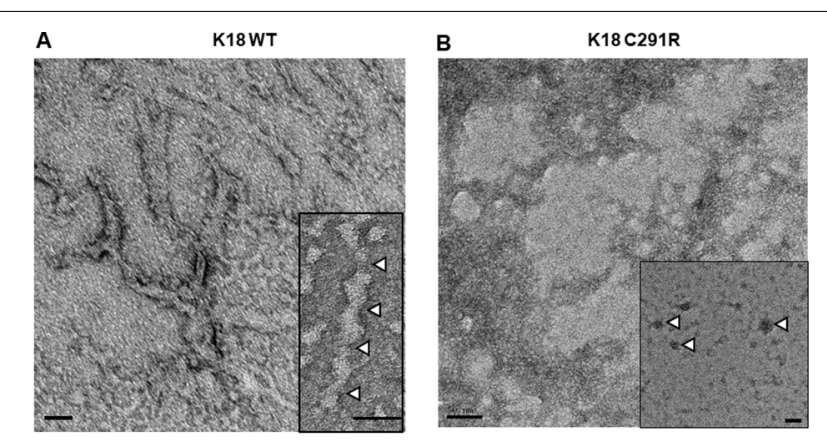

FIGURE 3 | Negative-stain transmission electron microscope (TEM) micrographs of aggregates of tau-K18 WT (A) and C291R (B) formed after treating $0.8 \mathrm{mg} / \mathrm{ml}$ of each construct with $0.4 \mathrm{mg} / \mathrm{ml}$ of heparin and incubating for $48 \mathrm{~h}$ at $37^{\circ} \mathrm{C}$ without shaking. Panel (A) shows fibrils with the inset highlighting the periodic twists of $\sim 50 \mathrm{~nm}$ (white arrowheads). Panel (B) shows amorphous aggregates, with the inset showing annular aggregates with defined outer structures (arrowheads). Scale bars $=50 \mathrm{~nm}$ for both main figures and insets.

wide and 150-300 nm long. On the contrary, tau-K18 C291R mostly formed amorphous structures without any regular shape or form (Figure 3B) in addition to annular aggregates 20-50 nm wide (Figure 3B, inset). These results suggest that the C291R mutation averts tau-K18 aggregation into fibrils, but rather favors the formation of non-fibrillar oligomers and amorphous assemblies.

\section{Tau-K18 C291R Aggregates Into Non-fibrillar Amorphous and Annular Structures in Native-State Conditions}

To exclude the possibility that tau-K18 C291R's aggregation into non-fibrillar structures was dependent on heparin induction, the experiment was repeated in native-state conditions by incubating both tau constructs in identical conditions as in Figure 3 except that no heparin was added. Aggregation was slower in this condition as anticipated (Karikari et al., 2019a). However, this approach allowed the fine mechanistic details of the process to be studied. AFM imaging revealed that tau-K18 WT monomers (Figure 4Ai) aggregated into granular oligomers (Figure 4Aii) and protofibril-like structures after $24 \mathrm{~h}$ (Figure 4Aii, inset). Clusters of aggregates identified at this stage (Figure 4Aii, whitefilled arrowheads) were likely to be off-pathway structures that did not follow amyloidogenic aggregation. More filamentous structures (protofibrils and short fibrils) were observed at $72 \mathrm{~h}$ through 216 h (Figures 4Aiii-v, black arrowheads). Bonafide fibrils of up to $7 \mu \mathrm{m}$ in length with paired helical filament (PHF) structure were recorded at $314 \mathrm{~h}$ (Figure 4Avi). This data shows that under careful conditions, tau-K18 WT can self-assemble into Alzheimer-like PHFs without the need for any external activation. Similar heparin-free aggregation studies have been performed for the recombinant form of a tau peptide (amino acid 297-391) that is a major component of PHF tau isolated from Alzheimer's disease brains (Al-Hilaly et al., 2017, 2018, 2019).

For tau-K18 C291R, small-sized structures at 0 h (Figure 4Bi) shifted towards more granular orientation with increased sizes
$(5-10 \mathrm{~nm})$ at $24 \mathrm{~h}$, with large amorphous structures over $3 \mu \mathrm{m}$ also evident at this stage (Figure 4Bii, inset). This coexistence of oligomers and amorphous aggregates was also observed at $72 \mathrm{~h}$ rather with increased oligomer sizes (Figure 4Biii). At $168 \mathrm{~h}$, several medium-sized amorphous structures $\sim 1 \mu \mathrm{m}$ became evident (Figure 4Biv, arrowheads). Closer inspection showed that these amorphous structures contained granular oligomers (Figure 5A, arrowheads), suggesting that some oligomers were either absorbed into the amorphous structures or the oligomers rearranged to form these large structures. Regular granular oligomers were also visible (Figure 5A, arrows), signifying the coexistence of two distinct types of aggregates i.e., granular oligomers and amorphous structures. At $216 \mathrm{~h}$, granular oligomers became less prominent but the medium-sized amorphous structures prevailed (Figure $4 \mathbf{B v}$ ). In addition, new structures with well-defined traces were recorded, which at higher resolution were made of various-sized amorphous clumps and groups of granular oligomers apparently in the process of forming amorphous clumps (Figure 5B, black arrowheads and arrows, respectively). Moreover, small ring-like annular structures made of granular oligomers were recorded beginning to take shape at $216 \mathrm{~h}$ (Figure 5B, white arrows). By $314 \mathrm{~h}$, larger ring-like structures including those enclosing granular oligomers were observed (Figures 4Bvi, 5C inset). The structures with well-defined traces formed at late time points were similar to the amorphous aggregates imaged in negative-stained conditions in Figure 3B. Together, these results indicate that the C291R mutation leads to drastic changes in the aggregation pathway and products of tau-K18, suggesting an altered conformational effect of the mutation.

\section{Tau-K18 C291R Forms Different Types of Protofibrils}

We characterized the C291R annular protofibrils formed at $314 \mathrm{~h}$ binning into four phenotypes, namely:

1. Spherical protofibrils: protofibrils consisting of granular oligomers arranged in a spherical manner. The sizes (diameter) of these aggregates ranged from 50 to $100 \mathrm{~nm}$, as well as up to over 1,000 $\mathrm{nm}$ (Figure 6A).

2. Spherical annular protofibrils encapsulating granular oligomers: in addition to being made of granular oligomers arranged in a spherical, ring-like structure, these aggregates consisted of single granular oligomer units dispersed within the enclosed structure (Figure 6B). These annular protofibrils were less frequently observed compared to the spherical ones above.

3. Ring-like annular but non-spherical protofibrils: These aggregates consisted of granular oligomers arranged in a non-spherical (often undefined) orientation (Figure 6C). The main similarity between this and the spherical protofibrils (phenotypes 1 and 2) is that they always formed a close-loop.

4. Linear protofibrils: these consisted of linearly arranged granular oligomers (Figure 6D), and differed from the other types of protofibrils by their arrangement compared to the ring-like structures of the others. 

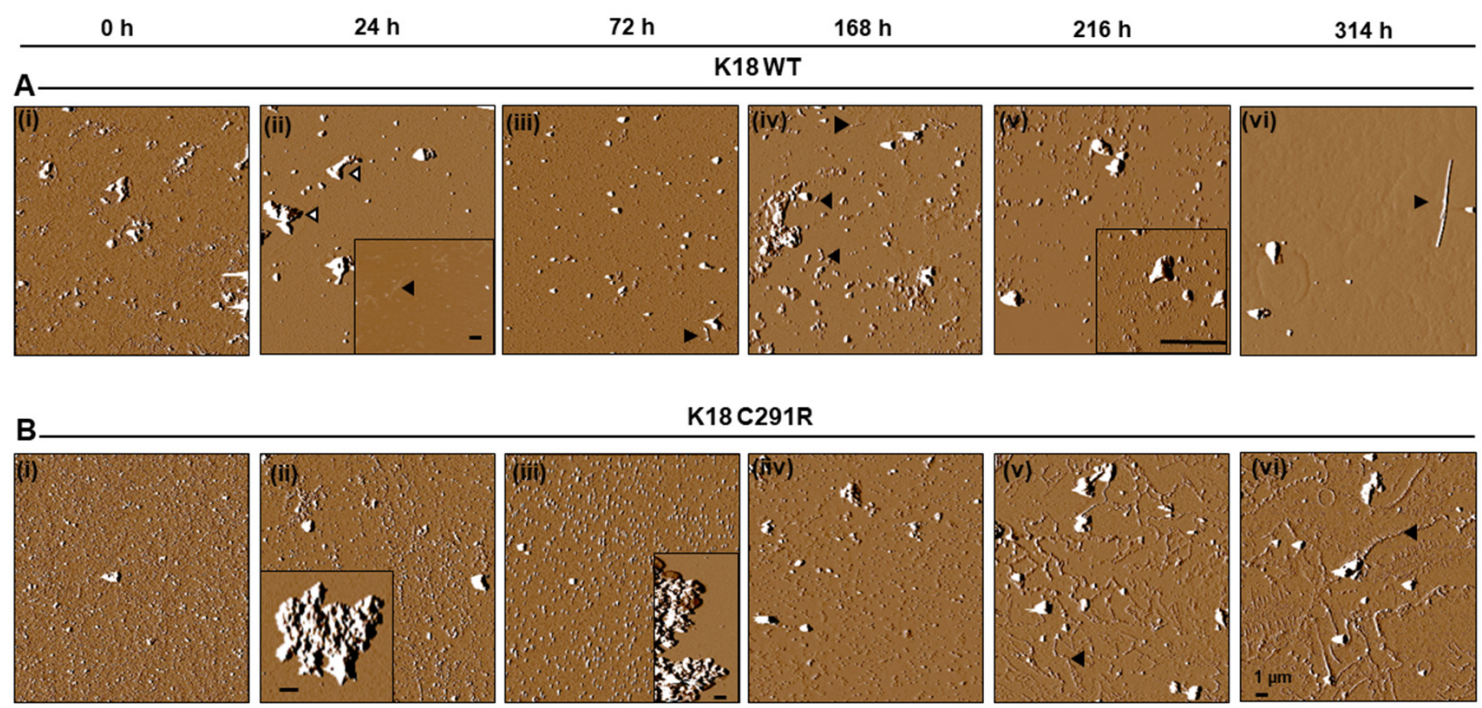

FIGURE 4 | Atomic force microscopy (AFM) images of tau-K18 WT (A) and C291R (B) aggregates formed over $314 \mathrm{~h}$ in native state conditions (incubation at $37^{\circ} \mathrm{C}$ without shaking). WT tau-K18 aggregated into fibrils at $314 \mathrm{~h}$ (arrowhead) via granular oligomers and protofibrils intermediates recorded at earlier time points. The C291R variant initially aggregated into granular oligomers, amorphous aggregates and later into different types of protofibrils. Scale bars = $1 \mu \mathrm{m}$ for all images. Further details about the intermediate structures may be found in the "Results" section.

$168 \mathrm{~h}$

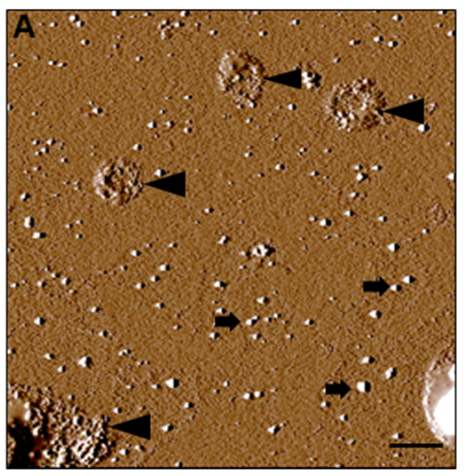

$216 \mathrm{~h}$

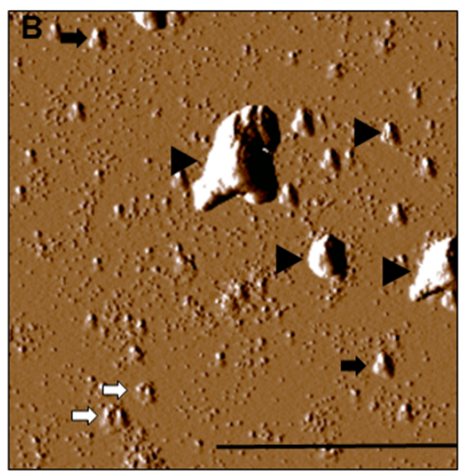

\section{$314 \mathrm{~h}$}

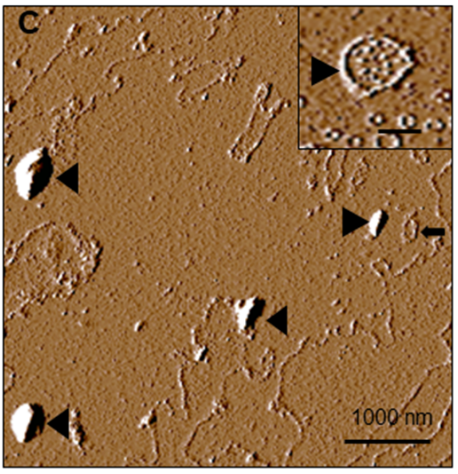

FIGURE 5 | Increased resolution AFM images of tau-K18 C291R at later stages of the native-state aggregation process. Aggregate structures formed at 168 h (A), $216 \mathrm{~h} \mathrm{(B)} \mathrm{and} 314 \mathrm{~h}$ (C) show granular oligomers (black arrows), amorphous aggregates (black arrowheads) and annular protofibrils including those encapsulating granular oligomers (inset in C). Scale bars $=1 \mu \mathrm{m}$.

The frequency distribution of the protofibrils at $314 \mathrm{~h}$, based on structure counts from AFM images from three experiments, can be found in Table 1.

\section{DISCUSSION}

In vitro biochemical studies provide critical molecular insights into potential disease-causing mechanisms of MAPT mutations identified in humans affected by specific tauopathies including effects on tau aggregation, phosphorylation, and microtubulebinding (Ghetti et al., 2015; Strang et al., 2019). However, little is known about MAPT mutations discovered more recently. In this study, we have provided the first biochemical characterization of the C291R tau variant, reported to have potentially pathogenic functions (Marshall et al., 2015). Cysteine-291 is a core component of CBD tau filament structure (Zhang et al., 2020), hence mutagenic changes are likely to have potential effects on aggregation properties. Our results demonstrate that the C291R mutation leads to a striking distinction in the aggregation mechanism of tau-K18 compared with the WT. Whilst WT tau-K18 underwent classical aggregation by progressively forming granular oligomers, protofibrils and fibrils, the C291R variant formed a mix of aggregates of three different major types: (1) granular oligomers, (2) amorphous 

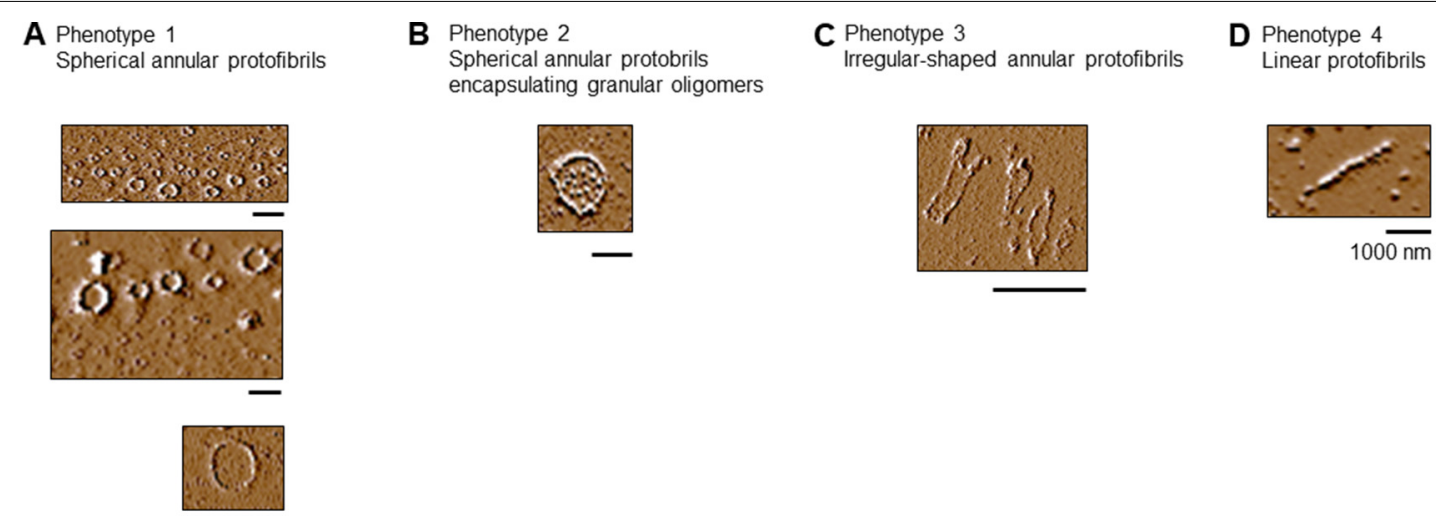

FIGURE 6 | Tau-K18 C291R forms linear and annular protofibrils of distinct phenotypes. (A) Classical spherical annular protofibrils, (B) spherical annular protofibrils encapsulating dispersed granular oligomers, (C) annular aggregates of undefined shape, and (D) classical linear protofibrils. Scale bars = $1 \mu \mathrm{m}$.

TABLE 1 | Frequency distribution of protofibrils phenotypes for tau-K18 C291R.
Phenotype

Spherical

Linear

Spherical encapsulating oligomers
Ring-like annular but non-spherical
Frequency (n, \%)

$223 / 329(67.8 \%)$

$56 / 329(17.0 \%)$

$42 / 329(12.8 \%)$

$8 / 329(2.4 \%)$ aggregates of various sizes and shapes, and (3) protofibrils-both linear and annular structures made of granular oligomers. It is noteworthy that these observations were made in both heparin-induced and native state aggregation reactions and imaged using two different methods-negative-stained TEM and label-free AFM. Importantly, we did not record obvious fibrillar aggregates for tau-K18 C291R but observed that in addition to regular linear protofibrils, this variant formed annular aggregates with unique features. Together, these lines of evidence are consistent with our hypothesis that the C291R mutation results in an altered aggregation pathway with aggregate conformers of distinct structural properties compared with WT tau-K18 aggregates.

The aggregation of tau-K18 C291R into a mix of granular oligomers, annular structures, and amorphous aggregates appears to be an off-pathway process with respect to fibril formation (Ding et al., 2002; Lasagna-Reeves et al., 2014). Key properties of this non-fibrillar pathway were clearly identified from our AFM analysis: the process begun with the formation of granular oligomers, which further polymerized into amorphous aggregates of various sizes and, at later time points, annular structures. The annular aggregates were made of granular oligomers arranged in a ring-like shape with defined outer structures. These properties easily differentiated them from the amorphous structures (see Figures 4B, 5C) and regular granular oligomers (Kayed et al., 2009). Due to their defined multi-oligomer presentation, annular aggregates are often referred to as annular protofibrils, pore-like structures or pore-forming aggregates, and have been widely characterized for amyloid-beta and alpha-synuclein (Ding et al., 2002; Lashuel et al., 2002; Kayed et al., 2009), and in a single report for tau (Lasagna-Reeves et al., 2014). In the case of tau, annular aggregates were identified, using TEM, AFM, and a conformation-specific antibody, in post mortem brains of dementia with Lewy body and progressive supranuclear palsy patients. Moreover, annular aggregates were reported from brain isolates of $\mathrm{P} 301 \mathrm{~L}$ tau-expressing mice, but not WT mice, suggesting an association with specific MAPT mutations (Lasagna-Reeves et al., 2014).

In the present study, we have demonstrated that a new, potentially pathogenic variant of tau, C291R, also forms annular aggregates in vitro in addition to linear protofibrils. Importantly, the annular aggregates shared the following striking similarities with those described previously for tau, amyloid-beta, and alphasynuclein: (1) their formation was preceded by spherical/granular oligomers; (2) the annular aggregates consisted of granular oligomers often arranged in a spherical fashion; (3) spherical annular aggregates were in a range of sizes; (4) annular aggregates were associated with a specific pathogenic mutation; and (5) pore-like annular protofibrils were clearly distinguishable from linear protofibrils which usually proceed to form fibrils (Ding et al., 2002; Lashuel et al., 2002, 2003; Lasagna-Reeves et al., 2014). In addition, two unique phenotypes of the tau-K18 C291R annular aggregates previously not described for an amyloidogenic protein/peptide were characterized, namely: (1) the spherically arranged granular oligomers that formed the annular aggregates occasionally enclosed small, single-unit granular oligomers within their larger ring structures (Figure 5C inset and Figure 6B); and (2) the ring-like shape of annular aggregates did not always have a spherical appearance. In fact, the shape was sometimes irregular and undefined (Figures 4Bv,vi, 5C, 6C). Together, we have demonstrated that the ring-like tau-K18 C291R aggregates possess key properties of annular protofibrils previously described for other amyloidogenic proteins. The new phenotypes presented to warrant further characterization in future studies.

Previous reports showed that substituting cysteine-291 with alanine (C291A) in WT tau forms affects the aggregation propensity, showing this reduces oligomer formation (Kim et al., 2015; Soeda et al., 2015; Al-Hilaly et al., 2017), 
demonstrating also that this does not prevent fibril formation (Barghorn and Mandelkow, 2002; Furukawa et al., 2011). Similarly, modifying cysteine-291 to serine (C291S) reduced its oligomerization competence (Kim et al., 2015). Together, these studies suggested that substituting cysteine-291 with Ala or Ser (both aliphatic amino acids) have similar effects. Moreover, we and others have shown that the C291A/C322A/I260C triplemodified K18 variant aggregates into both oligomers and fibrils (Kumar et al., 2014; Michel et al., 2014; Shammas et al., 2015; Karikari et al., 2019a,b). However, none of these modifications led to the formation of annular aggregates. Curiously, the C291A and C291S mutations both decreased aggregation competence in a manner distinct from the current C291R with a polar amino acid introduced, suggesting the choice of amino acid makes an important difference. Notably, the introduction of arginine at cysteine-291 had adverse effects on fibrillization processes but not oligomer formation as the C291R construct readily formed oligomers. We propose that the C291R mutation selectively impairs the conformational changes leading from oligomers to fibrils but not from monomers to oligomers. Cysteine residues in tau are surrounded by lysine residues whose basic charges enable disulfide bonding which is critical for aggregation (Lutolf et al., 2001). Removing one of the two cysteine residues in tau-K18 weakens its inter-molecular disulfide binding and hence aggregation tendency. The veracity of these hypothetical models need to be tested experimentally.

Although tau-K18 C291R did not form bona fide fibrils in the present study, we cannot rule out that the same construct can form fibrils under different experimental conditions (e.g., using different buffers and aggregation-inducing agents). Similar to our findings that linear and annular protofibrils of tau C291R were jointly present in the same reaction (see Figure 6), annular and fibrillar alpha-synuclein aggregates have been shown to co-exist in the same conditions (Shtilerman et al., 2002). Furthermore, tau P301L can form fibrils as well as annular aggregates in vitro and in vivo (Lasagna-Reeves et al., 2014), suggesting a high potential for co-existence.

A limitation of this study is that the proposed pathogenicity of the C291R mutation has not been confirmed by neuropathological examination. Therefore, the mutation and its functional properties described in this manuscript cannot be presently linked to CBD or any other form of tauopathy. Nonetheless, the results provide insights into the functional and structural properties of this novel mutation and sheds new light on the biochemical basis of tau aggregation. Moreover, reported differences in aggregate structures of human brain-derived tau and recombinant tau aggregated in vitro by inducing with heparin (Zhang et al., 2019) may limit the

\section{REFERENCES}

Al-Hilaly, Y. K., Foster, B. E., Biasetti, L., Lutter, L., Pollack, S. J., Rickard, J. E., et al. (2019). Tau (297-391) forms filaments that structurally mimic the core of paired helical filaments in Alzheimer's disease brain. FEBS Lett. doi: 10.1002/1873-3468.13675 [Epub ahead of print].

Al-Hilaly, Y. K., Pollack, S. J., Rickard, J. E., Simpson, M., Raulin, A.-C., Baddeley, T., et al. (2018). Cysteine-independent inhibition of Alzheimer's utility of the findings presented in this study. However, a more recent study showed close similarities between brain-derived tau filaments and those formed by the amino acid 297-391 fragment self-assembled in vitro without any external inducing agents (Al-Hilaly et al., 2019). Given that the tau-K18 C291R construct studied herein could also self-aggregate and contains about half of the tau 297-391 sequence, the findings of this study are likely to have potential implications for future in vivo and neuropathological studies.

\section{CONCLUSION}

We have presented the first biochemical characterization of tau C291R, covering its secondary structure, step-wise aggregation stages, and conformational properties. A novel, significant finding is that tau C291R forms annular, pore-like aggregates, the second time this phenomenon has been described for a tau construct after tau P301L. Importantly, the C291R annular aggregates share major properties described for such structures formed by amyloid-beta, alpha-synuclein and tau P301L but also has two unique features previously unknown: (1) spherically arranged granular oligomers that enclose small, single-unit granular oligomers; and (2) non-spherical ring-like annular aggregates. These findings, and their further characterization in future studies, should pave the way for further studies into the C291R tau variant and how the annular aggregates it forms could provide new insights into the aggregation process of tau.

\section{DATA AVAILABILITY STATEMENT}

All datasets generated for this study are included in the article.

\section{AUTHOR CONTRIBUTIONS}

TK and KM conceived and co-ordinated the study and wrote the manuscript. TK and RT performed the experiments and analyzed the data. All authors critically revised and agreed on the final manuscript.

\section{FUNDING}

TK acknowledges a joint studentship from the University of Warwick Chancellor's Scholarship and the Biotechnology and Biological Sciences Research Council (BBSRC) grant number BB/J014532/1, through the Midlands Integrative Biosciences Training Partnership. The funders had no role in study design, data collection and analysis, decision to publish, or preparation of the manuscript.

disease-like paired helical filament assembly by leuco-methylthioninium (LMT). J. Mol. Biol. 430, 4119-4131. doi: 10.1016/j.jmb.2018.08.010

Al-Hilaly, Y. K., Pollack, S. J., Vadukul, D., Citossi, F., Rickard, J. E., Simpson, M., et al. (2017). Alzheimer's disease-like paired helical filament assembly from truncated tau protein is independent of disulphide cross-linking. J. Mol. Biol. 429, 3650-3665. doi: 10.1016/j.jmb.2017.09.007

Andreadis, A. (2005). Tau gene alternative splicing: expression patterns, regulation and modulation of function in normal brain and neurodegenerative 
diseases. Biochim. Biophys. Acta 1739, 91-103. doi: 10.1016/j.bbadis.2004. 08.010

Andreadis, A., Brown, W. M., and Kosik, K. S. (1992). Structure and novel exons of the human tau gene. Biochemistry 31, 10626-10633. doi: 10.1021/bi00 $158 \mathrm{a} 027$

Barghorn, S., and Mandelkow, E. (2002). Toward a unified scheme for the aggregation of tau into Alzheimer paired helical filaments. Biochemistry 41, 14885-14896. doi: 10.1021/bi026469j

Barghorn, S., Zheng-Fischhöfer, Q., Ackmann, M., Biernat, J., von Bergen, M., Mandelkow, E.-M., et al. (2000). Structure, microtubule interactions and paired helical filament aggregation by tau mutants of frontotemporal dementias $\dagger$. Biochemistry 39, 11714-11721. doi: 10.1021/bi000850r

Bhattacharya, K., Rank, K. B., Evans, D. B., and Sharma, S. K. (2001). Role of cysteine-291 and cysteine-322 in the polymerization of human tau into Alzheimer-like filaments. Biochem. Biophys. Res. Commun. 285, 20-26. doi: 10.1006/bbrc. 2001.5116

Chen, H., Liu, S., Li, S., Chen, J., Ni, J., and Liu, Q. (2018). Blocking the thiol at cysteine-322 destabilizes tau protein and prevents its oligomer formation. ACS Chem. Neurosci. 9, 1560-1565. doi: 10.1021/acschemneuro. 8 b00003

Cisek, K., Cooper, G. L., Huseby, C. J., and Kuret, J. (2014). Structure and mechanism of action of tau aggregation inhibitors. Curr. Alzheimer Res. 11, 918-927. doi: 10.2174/1567205011666141107150331

Cohen, T. J., Friedmann, D., Hwang, A. W., Marmorstein, R., and Lee, V. M. Y. (2013). The microtubule-associated tau protein has intrinsic acetyltransferase activity. Nat. Struct. Mol. Biol. 20, 756-762. doi: 10.1038/nsmb.2555

Combs, B., and Gamblin, T. C. (2012). FTDP-17 tau mutations induce distinct effects on aggregation and microtubule interactions. Biochemistry 51, 8597-8607. doi: 10.1021/bi3010818

Crowe, A., James, M. J., Lee, V. M.-Y., Smith, A. B., Trojanowski, J. Q., Ballatore, C., et al. (2013). Aminothienopyridazines and methylene blue affect tau fibrillization via cysteine oxidation. J. Biol. Chem. 288, 11024-11037. doi: $10.1074 /$ jbc.m112.436006

Ding, T. T., Lee, S.-J., Rochet, J.-C., and Lansbury, P. T. (2002). Annular $\alpha-$ synuclein protofibrils are produced when spherical protofibrils are incubated in solution or bound to brain-derived membranes. Blood 41, 10209-10217. doi: 10.1182/blood-2015-12-688838

Furukawa, Y., Kaneko, K., and Nukina, N. (2011). Tau protein assembles into isoform- and disulfide-dependent polymorphic fibrils with distinct structural properties. J. Biol. Chem. 286, 27236-27246. doi: 10.1074/jbc.m111. 248963

Ghetti, B., Oblak, A. L., Boeve, B. F., Johnson, K. A., Dickerson, B. C., and Goedert, M. (2015). Frontotemporal dementia caused by microtubuleassociated protein tau gene (MAPT) mutations: a chameleon for neuropathology and neuroimaging. Neuropathol. Appl. Neurobiol. 41, 24-46. doi: 10.1111/nan.12213

Goedert, M., and Jakes, R. (2005). Mutations causing neurodegenerative tauopathies. Biochim. Biophys. 1739, 240-250. doi: 10.1016/j.bbadis. 2004 . 08.007

Goedert, M., Spillantini, M. G., Potier, M. C., Ulrich, J., and Crowther, R. A. (1989). Cloning and sequencing of the cDNA encoding an isoform of microtubuleassociated protein tau containing four tandem repeats: differential expression of tau protein mRNAs in human brain. EMBO J. 8, 393-399. doi: 10.1002/j. 1460-2075.1989.tb03390.x

Goedert, M., Wischik, C. M., Crowther, R. A., Walker, J. E., and Klug, A. (1988). Cloning and sequencing of the cDNA encoding a core protein of the paired helical filament of Alzheimer disease: identification as the microtubule-associated protein tau. Proc. Natl. Acad. Sci. U S A 85, 4051-4055. doi: 10.1073/pnas.85.11.4051

Hill, E., Karikari, T. K., Moffat, K. G., Richardson, M. J. E., and Wall, M. J. (2019). Introduction of tau oligomers into cortical neurons alters action potential dynamics and disrupts synaptic transmission and plasticity. eNeuro 6:ENEURO.0166-19.2019. doi: 10.1523/eneuro.0166-19.2019

Huseby, C. J., Bundschuh, R., and Kuret, J. (2019). The role of annealing and fragmentation in human tau aggregation dynamics. J. Biol. Chem. 294, 4728-4737. doi: 10.1074/jbc.ra118.006943

Karikari, T. K., Nagel, D. A., Grainger, A., Clarke-Bland, C., Crowe, J., Hill, E. J., et al. (2019a). Distinct conformations, aggregation and cellular internalization of different tau strains. Front. Cell. Neurosci. 13:296. doi: 10.3389/fncel.2019. 00296

Karikari, T. K., Nagel, D. A., Grainger, A., Clarke-Bland, C., Hill, E. J., and Moffat, K. G. (2019b). Preparation of stable tau oligomers for cellular and biochemical studies. Anal. Biochem. 566, 67-74. doi: 10.1016/j.ab.2018. 10.013

Karikari, T. K., Turner, A., Stass, R., Lee, L. C. Y., Wilson, B., Nagel, D. A., et al. (2017). Expression and purification of tau protein and its frontotemporal dementia variants using a cleavable histidine tag. Protein Expr. Purif. 130, 44-54. doi: 10.1016/j.pep.2016.09.009

Kayed, R., Pensalfini, A., Margol, L., Sokolov, Y., Sarsoza, F., Head, E., et al. (2009). Annular protofibrils are a structurally and functionally distinct type of amyloid oligomer. J. Biol. Chem. 284, 4230-4237. doi: 10.1074/jbc.M808 591200

Kim, D., Lim, S., Haque, M. D. M., Ryoo, N., Hong, H. S., Rhim, H., et al. (2015). Identification of disulfide cross-linked tau dimer responsible for tau propagation. Sci. Rep. 5:15231. doi: 10.1038/srep15231

Kumar, S., Tepper, K., Kaniyappan, S., Biernat, J., Wegmann, S., Mandelkow, E.M., et al. (2014). Stages and conformations of the tau repeat domain during aggregation and its effect on neuronal toxicity. J. Biol. Chem. 289, 20318-20332. doi: $10.1074 /$ jbc.m114.554725

Lasagna-Reeves, C. A., Sengupta, U., Castillo-Carranza, D., Gerson, J. E., Guerrero-Munoz, M., Troncoso, J. C., et al. (2014). The formation of tau pore-like structures is prevalent and cell specific: possible implications for the disease phenotypes. Acta Neuropathol. Commun. 2:56. doi: 10.1186/20515960-2-56

Lashuel, H. A., Hartley, D. M., Petre, B. M., Wall, J. S., Simon, M. N., Walz, T., et al. (2003). Mixtures of wild-type and a pathogenic (E22G) form of Abeta40 in vitro accumulate protofibrils, including amyloid pores. J. Mol. Biol. 332, 795-808. doi: 10.1016/s0022-2836(03)00927-6

Lashuel, H. A., Hartley, D., Petre, B. M., Walz, T., and Lansbury, P. T.Jr. (2002). Amyloid pores from pathogenic mutations. Nature 418:291. doi: $10.1038 / 418291 \mathrm{a}$

Liu, F., and Gong, C.-X. (2008). Tau exon 10 alternative splicing and tauopathies. Mol. Neurodegener. 3:8. doi: 10.1186/1750-1326-3-8

Lutolf, M. P., Tirelli, N., Cerritelli, S., Cavalli, L., and Hubbell, J. A. (2001). Systematic modulation of michael-type reactivity of thiols through the use of charged amino acids. Bioconjug. Chem. 12, 1051-1056. doi: 10.1021/bc0 $15519 \mathrm{e}$

Marshall, C. R., Guerreiro, R., Thust, S., Fletcher, P., Rohrer, J. D., and Fox, N. C. (2015). A novel MAPT mutation causing corticobasal syndrome led by progressive apraxia of speech. J. Alzheimers. Dis. 48, 923-926. doi: 10.3233/jad150477

Michel, C. H., Kumar, S., Pinotsi, D., Tunnacliffe, A., George-Hyslop, P. S., Mandelkow, E., et al. (2014). Extracellular monomeric tau protein is sufficient to initiate the spread of tau protein pathology. J. Biol. Chem. 289, 956-967. doi: 10.1074/jbc.M113.515445

Neve, R. L., Harris, P., Kosik, K. S., Kurnit, D. M., and Donlon, T. A. (1986). Identification of cDNA clones for the human microtubule-associated protein tau and chromosomal localization of the genes for tau and microtubule-associated protein 2. Brain Res. 387, 271-280. doi: 10.1016/0169$328 \mathrm{x}(86) 90033-1$

Schweers, O., Mandelkow, E. M., Biernat, J., and Mandelkow, E. (1995). Oxidation of cysteine-322 in the repeat domain of microtubule-associated protein tau controls the in vitro assembly of paired helical filaments. Proc. Natl. Acad. Sci. U S A 92, 8463-8467. doi: 10.1073/pnas.92.18.8463

Shammas, S. L., Garcia, G. A., Kumar, S., Kjaergaard, M., Horrocks, M. H., Shivji, N., et al. (2015). A mechanistic model of tau amyloid aggregation based on direct observation of oligomers. Nat. Commun. 6:7025. doi: $10.1038 /$ ncomms 8025

Shtilerman, M. D., Ding, T. T., and Lansbury, P. T. (2002). Molecular crowding accelerates fibrillization of alpha-synuclein: could an increase in the cytoplasmic protein concentration induce Parkinson's disease? Biochemistry 41, 3855-3860. doi: 10.1021/bi0120906

Soeda, Y., Yoshikawa, M., Almeida, O. F. X., Sumioka, A., Maeda, S., Osada, H., et al. (2015). Toxic tau oligomer formation blocked by capping of cysteine residues with 1,2-dihydroxybenzene groups. Nat. Commun. 6:10216. doi: $10.1038 /$ ncomms 10216 
Strang, K. H., Golde, T. E., and Giasson, B. I. (2019). MAPT mutations, tauopathy and mechanisms of neurodegeneration. Lab. Invest. 99, 912-928. doi: 10.1038/s41374-019-0197-x

Zhang, W., Falcon, B., Murzin, A. G., Fan, J., Crowther, R. A., Goedert, M., et al. (2019). Heparin-induced tau filaments are polymorphic and differ from those in Alzheimer's and Pick's diseases. eLife 8:e43584. doi: 10.7554/eLife. 43584

Zhang, W., Tarutani, A., Newell, K. L., Murzin, A. G., Matsubara, T., Falcon, B., et al. (2020). Novel tau filament fold in corticobasal degeneration. Nature doi: 10.1038/s41586-020-2043-0 [Epub ahead of print].
Conflict of Interest: The authors declare that the research was conducted in the absence of any commercial or financial relationships that could be construed as a potential conflict of interest.

Copyright (c) 2020 Karikari, Thomas and Moffat. This is an open-access article distributed under the terms of the Creative Commons Attribution License (CC BY). The use, distribution or reproduction in other forums is permitted, provided the original author(s) and the copyright owner(s) are credited and that the original publication in this journal is cited, in accordance with accepted academic practice. No use, distribution or reproduction is permitted which does not comply with these terms. 